TRANSACTIONS OF THE

AMERICAN MATHEMATICAL SOCIETY

Volume 350, Number 5, May 1998, Pages 2071-2085

S 0002-9947(98)01919-9

\title{
LOCAL SPECTRA AND INDIVIDUAL STABILITY OF UNIFORMLY BOUNDED $C_{0}$-SEMIGROUPS
}

\author{
CHARLES J. K. BATTY, JAN VAN NEERVEN, AND FRANK RÄBIGER
}

\begin{abstract}
We study the asymptotic behaviour of individual orbits $T(\cdot) x$ of a uniformly bounded $C_{0}$-semigroup $\{T(t)\}_{t}>0$ with generator $A$ in terms of the singularities of the local resolvent $(\lambda-A)^{-1} x$ on the imaginary axis. Among other things we prove individual versions of the Arendt-Batty-Lyubich-Vũ theorem and the Katznelson-Tzafriri theorem.
\end{abstract}

\section{INTRODUCTION}

A $C_{0}$-semigroup $\mathbf{T}=\{T(t)\}_{t>0}$ on a Banach space $X$ is said to be uniformly stable if $\lim _{t \rightarrow \infty}\|T(t) x\|=0$ for all $x \in X$. In 1988, Arendt and Batty [1] and Lyubich and Vũ [17] proved the following result.

Theorem A. Let $\mathbf{T}$ be a uniformly bounded $C_{0}$-semigroup on a Banach space $X$, with generator $A$. If

(i) $\sigma(A) \cap i \mathbb{R}$ is countable,

(ii) $\sigma_{p}\left(A^{*}\right) \cap i \mathbb{R}=\emptyset$,

then $\mathbf{T}$ is uniformly stable.

Here, $\sigma(A)$ denotes the spectrum of $A$ and $\sigma_{p}\left(A^{*}\right)$ is the point spectrum of the adjoint $A^{*}$ of $A$. Examples show that none of the assumptions can be relaxed, so this result is optimal in some sense. A little later Esterle, Strouse and Zouakia [10] and Vu [21] each gave a proof of the following continuous parameter version of the Katznelson-Tzafriri theorem (see [15] for the discrete case).

Theorem B. Let $\mathbf{T}$ be a uniformly bounded $C_{0}$-semigroup on a Banach space $X$, with generator $A$. If $f \in L^{1}\left(\mathbb{R}_{+}\right)$is of spectral synthesis with respect to i $\sigma(A) \cap \mathbb{R}$, then $\lim _{t \rightarrow \infty}\|T(t) \hat{f}(\mathbf{T})\|=0$.

Here, $\hat{f}(\mathbf{T})$ is the bounded operator on $X$ defined by

$$
\hat{f}(\mathbf{T}) x:=\int_{0}^{\infty} f(t) T(t) x d t, \quad x \in X .
$$

Received by the editors February 12, 1996 and, in revised form, September 6, 1996.

1991 Mathematics Subject Classification. Primary 47D03.

Key words and phrases. Laplace transform, singular set, countable, $C_{0}$-semigroup, stability, local spectrum, orbit.

The work on this paper was done during a two-year stay at the University of Tübingen. Support by an Individual Fellowship from the Human Capital and Mobility Programme of the European Community is gratefully acknowledged. I warmly thank Professor Rainer Nagel and the members of his group for their hospitality (second author). It is part of a research project supported by the Deutsche Forschungsgemeinschaft DFG (third author). Work in Oxford was also supported by an EPSRC Visiting Fellowship Research Grant (first and third authors).

(c)1998 American Mathematical Society 
This result is different from the first in that there are no restrictions on the spectrum of $A$, but stability is only obtained for certain orbits. For the history of these and related results we refer to [3].

In this paper we address the problem whether individual versions of the above theorems hold. Such versions can be found e.g. in [1], [4], [8], [9], however there the assumed spectral conditions are all global. Our main idea is to relate the asymptotic behaviour of an individual orbit $T(\cdot) x$ to the behaviour of the local resolvent $R(\lambda, A) x$ near the imaginary axis; here $R(\lambda, A)=(\lambda-A)^{-1}$ is the resolvent of $A$ at the point $\lambda$. More precisely, we define the local unitary spectrum $\sigma_{u}(A, x)$ of $x$ to be the set of points $\lambda_{0} \in i \mathbb{R}$ to which $\lambda \mapsto R(\lambda, A) x$ cannot be extended holomorphically, and study the relationship between the asymptotic behaviour of $T(\cdot) x$ and the local unitary spectrum of $x$.

It turns out that, for a given $x \in X$, assumption (i) in the Arendt-Batty-LyubichVũ theorem can be relaxed to countability of the local unitary spectrum of $x$, in which case we have $\lim _{t \rightarrow \infty}\|T(t) x\|=0$. This is proved in Section 3, where it is also shown how to localize assumption (ii) to a certain ergodic condition on the orbit of $x$.

As a consequence of our individual Arendt-Batty-Lyubich-Vũ theorem we obtain the following stability result: a uniformly bounded $C_{0}$-semigroup on $X$ is uniformly stable if $\sigma_{p}\left(A^{*}\right)=\emptyset$ and there exists a dense subspace $Z \subset X$ such that the local unitary spectrum of each $z \in Z$ is countable. This generalizes results of Huang [12] and Batty [5], where among other things it was assumed that the local unitary spectrum is contained in a fixed countable set for all $z \in Z$. Huang [12] conjectured that a result of this type might characterize uniform stability. In Section 4 we refute this conjecture by providing an example of a uniformly stable semigroup with the property that the local unitary spectrum of each non-zero $x \in X$ is all of $i \mathbb{R}$.

Also the Katznelson-Tzafriri theorem admits an individual version. Assuming that $\mathbf{T}$ is uniformly bounded and $f$ is of spectral synthesis with respect to $i \sigma_{u}(A, x)$, we show that $\lim _{t \rightarrow \infty}\|T(t) \hat{f}(\mathbf{T}) x\|=0$. This is proved in Section 5 .

Our individual stability results depend on a theorem, proved in Section 2, which shows that the local unitary spectrum does not enlarge when passing to elements in the closed $\mathbf{T}$-invariant subspace generated by $x$, provided the semigroup is isometric. The proof of this depends on a construction which was already exploited in [1] and [5] and goes back to Carleman.

In Sections 6 and 7 we apply our results to obtain an individual version of a result of Lyubich and Vũ [22] on almost periodicity of $C_{0}$-semigroups and of a recent quantitative stability result of Batty, Brzeźniak and Greenfield [6].

In [7], we show that the results of this paper can be extended to individual bounded, uniformly continuous orbits of an arbitrary (possibly unbounded) $C_{0^{-}}$ semigroup. That this is possible is a non-trivial fact and depends on another analytic extension result for local resolvents, by means of which the unbounded case is reduced to the bounded case. Moreover we apply our theory to derive a new Tauberian theorem for the Laplace transform of certain bounded $X$-valued functions.

\section{The MAIN EXTENSION THEOREM}

In this paper $\mathbf{T}=\{T(t)\}_{t \geq 0}$ always denotes a uniformly bounded $C_{0}$-semigroup on a Banach space $X$ and $A$ its generator. If $x \in X$, then the map 
$R:\{\operatorname{Re} \lambda>0\} \rightarrow X: \lambda \mapsto R(\lambda, A) x$ is holomorphic. The local unitary spectrum $\sigma_{u}(A, x)$ of $x$ is the set of points $\lambda \in i \mathbb{R}$ to which $R$ cannot be extended holomorphically. Clearly $\sigma_{u}(A, x) \subset \sigma(A) \cap i \mathbb{R}$, and an easy application of the uniform boundedness principle yields

$$
\sigma(A) \cap i \mathbb{R}=\bigcup_{x \in X} \sigma_{u}(A, x) .
$$

For a given $x \in X$ let $X_{x}$ be the closed linear span of the orbit $\{T(t) x: t \geq 0\}$. Then $X_{x}$ is $\mathbf{T}$-invariant and we denote by $\mathbf{T}_{x}=\left\{T_{x}(t)\right\}_{t \geq 0}$ the restriction of $\mathbf{T}$ to $X_{x}$ and by $A_{x}$ the generator of $\mathbf{T}_{x}$.

For our main extension theorem we need the following lemma based on [17, Lemma]. Recall that a bounded operator $T$ on $X$ is an isometry if $\|T x\|=\|x\|$ for all $x \in X$.

Lemma 2.1. Let $\mathbf{T}$ be a $C_{0}$-semigroup of isometries on a Banach space $X$, with generator $A$. Let $x \in X$ and let $F$ be a holomorphic extension of $\lambda \mapsto R(\lambda, A) x$ to a connected neighbourhood $V$ of $\{\operatorname{Re} \lambda \geq 0\} \backslash \sigma_{u}(A, x)$. Then $\|F(\lambda)\| \leq|\operatorname{Re} \lambda|^{-1}\|x\|$ for all $\lambda \in V \backslash i \mathbb{R}$.

Proof. From the identity

$$
e^{-\lambda t} T(t) R(\lambda, A) x=R(\lambda, A) x-\int_{0}^{t} e^{-\lambda s} T(s) x d s,
$$

valid for $\operatorname{Re} \lambda>0$, and the uniqueness theorem for analytic functions we have

$$
e^{-\lambda t} T(t) F(\lambda)=F(\lambda)-\int_{0}^{t} e^{-\lambda s} T(s) x d s, \quad \lambda \in V .
$$

Since $\mathbf{T}$ is isometric, it follows that

$$
\begin{aligned}
e^{-\operatorname{Re} \lambda t}\|F(\lambda)\| & =e^{-\operatorname{Re} \lambda t}\|T(t) F(\lambda)\| \\
& \leq\|F(\lambda)\|+\int_{0}^{t} e^{-\operatorname{Re} \lambda s}\|T(s) x\| d s \\
& =\|F(\lambda)\|+\left(\int_{0}^{t} e^{-\operatorname{Re} \lambda s} d s\right)\|x\| \\
& =\|F(\lambda)\|+\frac{e^{-\operatorname{Re} \lambda t}-1}{-\operatorname{Re} \lambda}\|x\| .
\end{aligned}
$$

This yields the desired inequality for all $\lambda \in V$ with negative real part. For $\operatorname{Re} \lambda>0$ the conclusion follows by estimating the Laplace integral $R(\lambda, A) x=$ $\int_{0}^{\infty} e^{-\lambda s} T(s) x d s$.

With the notation introduced above we obtain the following extension theorem.

Theorem 2.2. Let $\mathbf{T}$ be a $C_{0}$-semigroup of isometries on a Banach space $X$, with generator $A$. For each $x \in X$ there exists a connected neighbourhood $W$ of $\{\operatorname{Re} \lambda \geq$ $0\} \backslash \sigma_{u}(A, x)$ such that $\lambda \mapsto R(\lambda, A) z$ admits an $X_{x}$-valued holomorphic extension to $W$ for all $z \in X_{x}$. In particular $\sigma\left(A_{x}\right) \cap i \mathbb{R}=\sigma_{u}(A, x)$.

Proof. Let $V$ be a connected neighbourhood of $\{\operatorname{Re} \lambda \geq 0\} \backslash \sigma_{u}(A, x)$ such that $\lambda \mapsto R(\lambda, A) x$ admits a holomorphic extension $F$ to $V$. Denote by $Z$ the linear span of the orbit $\{T(t) x: t \geq 0\}$. Fix $z \in X_{x}=\bar{Z}$ and choose a sequence $\left(z_{n}\right) \subset Z$ with $z_{n} \rightarrow z$. Since $T(t) F(\lambda)$ is a holomorphic extension of $R(\lambda, A) T(t) x$ to $V$ for each $t \geq 0$ we see that each $R(\lambda, A) z_{n}$ admits a holomorphic extension $F_{n}(\lambda)$ to $V$. 
For $\lambda_{0} \in i \mathbb{R} \backslash \sigma_{u}(A, x)$ we choose $r\left(\lambda_{0}\right)>0$ such that the closure of the open ball $B\left(\lambda_{0}, r\left(\lambda_{0}\right)\right)$ of radius $r\left(\lambda_{0}\right)$ and centre $\lambda_{0}$ is contained in $V$. Let $B$ denote the union of all balls $B\left(\lambda_{0}, \frac{1}{2} r\left(\lambda_{0}\right)\right), \lambda_{0} \in i \mathbb{R} \backslash \sigma_{u}(A, x)$, and put $W=\{\operatorname{Re} \lambda>0\} \cup B$. Then $W \subset V$ and $W$ is a connected neighbourhood of $\{\operatorname{Re} \lambda \geq 0\} \backslash \sigma_{u}(A, x)$. In order to prove that $R(\lambda, A) z$ extends holomorphically to $W$, it suffices to show that it extends to each $B\left(\lambda_{0}, \frac{1}{2} r\left(\lambda_{0}\right)\right)$.

So let $\lambda_{0} \in i \mathbb{R} \backslash \sigma_{u}(A, x)$ and $r=r\left(\lambda_{0}\right)$ as above be fixed. By rescaling $\mathbf{T}$, we may assume that $\lambda_{0}=0$. We claim that the functions $F_{n}$ are bounded on $B\left(0, \frac{1}{2} r\right)$, uniformly in $n$. Once this has been shown, from the fact that $R(\lambda, A) z_{n} \rightarrow R(\lambda, A) z$ for $\operatorname{Re} \lambda>0$ it follows by Vitali's theorem that the functions $F_{n}$ converge, uniformly on compacta, to a holomorphic function $F$ on $B\left(0, \frac{1}{2} r\right)$. This $F$ is the desired extension of $R(\lambda, A) z$.

Define the continuous functions $f_{n}: \overline{B(0, r)} \rightarrow X$ by

$$
f_{n}(\lambda):=\left(1+\frac{\lambda^{2}}{r^{2}}\right) F_{n}(\lambda) .
$$

Each $f_{n}$ is holomorphic in $B(0, r)$. By virtue of Lemma 2.1, for $\lambda=r e^{i \theta} \in$ $\partial B(0, r) \backslash i \mathbb{R}$ we have

$$
\left\|f_{n}(\lambda)\right\| \leq\left|1+\frac{\lambda^{2}}{r^{2}}\right| \cdot \frac{1}{|\operatorname{Re} \lambda|}\left\|z_{n}\right\|=\left|1+e^{2 i \theta}\right| \cdot \frac{1}{r|\cos \theta|}\left\|z_{n}\right\|=\frac{2}{r}\left\|z_{n}\right\| .
$$

Therefore, by the maximum modulus theorem,

$$
\sup _{\lambda \in B(0, r)}\left\|f_{n}(\lambda)\right\| \leq \frac{2}{r}\left\|z_{n}\right\|
$$

It follows that

$$
\begin{aligned}
\sup _{n} \sup _{\lambda \in B\left(0, \frac{r}{2}\right)}\left\|F_{n}(\lambda)\right\| & =\sup _{n} \sup _{\lambda \in B\left(0, \frac{r}{2}\right)} \frac{r^{2}}{\left|r^{2}+\lambda^{2}\right|}\left\|f_{n}(\lambda)\right\| \\
& \leq \sup _{n} \sup _{\lambda \in B\left(0, \frac{r}{2}\right)} \frac{2 r}{\left|r^{2}+\lambda^{2}\right|}\left\|z_{n}\right\| \leq \frac{8}{3 r} \sup _{n}\left\|z_{n}\right\| .
\end{aligned}
$$

This proves the claim.

So far we have shown that for $z \in X_{x}$ the local resolvent $R(\lambda, A) z$ has a holomorphic extension $F_{z}$ to $W$. If $\operatorname{Re} \lambda>0$ then the resolvent of $A$ and $A_{x}$ can be represented as the Laplace transform of $\mathbf{T}$ and $\mathbf{T}_{x}$, respectively, and hence $R(\lambda, A) z=R\left(\lambda, A_{x}\right) z$. Let $q: X \rightarrow X / X_{x}$ be the quotient map. Then $q F_{z}(\lambda)$ is a holomorphic extension of $q R(\lambda, A) z$ and $q F_{z}(\lambda)=0$ for $\operatorname{Re} \lambda>0$. Thus $q F_{z}(\lambda)=0$ on $W$, i.e. $F_{z}$ is $X_{x}$-valued on $W$. Hence $F_{z}$ is a holomorphic extension of $R\left(\lambda, A_{x}\right) z$. This implies

$$
\sigma_{u}(A, x)=\sigma_{u}\left(A_{x}, x\right) \subset \bigcup_{z \in X_{x}} \sigma_{u}\left(A_{x}, z\right) \subset \sigma_{u}(A, x) .
$$

Thus $\sigma\left(A_{x}\right) \cap i \mathbb{R}=\bigcup_{z \in X_{x}} \sigma_{u}\left(A_{x}, z\right)=\sigma_{u}(A, x)$.

Remarks. (a) The last part of the proof shows that for a $C_{0}$-semigroup $\mathbf{T}$ on $X$ and $x \in X$ one always has $\sigma_{u}(A, x)=\sigma_{u}\left(A_{x}, x\right)$.

(b) The theorem cannot be extended to contraction semigroups; this is shown by a counterexample in [7] for the left translation semigroup on $X=C_{0}\left(\mathbb{R}_{+}\right)$. 


\section{An individual Arendt-Batty-Lyubich-VŨ theorem}

In this section we present an individual version of the Arendt-Batty-Lyubich-Vũ theorem. First we 'localize' the conditions imposed in Theorem A and then we show that from the local conditions we obtain convergence to 0 of an individual orbit.

If $\mathbf{T}$ is a uniformly bounded $C_{0}$-semigroup on $X$, with generator $A$, and if $x \in X$ is given, then it is natural to replace the countability condition on $\sigma(A) \cap i \mathbb{R}$ by the countability of $\sigma_{u}(A, x)$. In order to localize the condition $\sigma_{p}\left(A^{*}\right) \cap i \mathbb{R}=\emptyset$ we observe that convergence of $T(t) x$ to 0 as $t \rightarrow \infty$ also follows if we apply Theorem A to the restriction $\mathbf{T}_{x}$ of $\mathbf{T}$ to $X_{x}$. Thus we may replace the condition $\sigma_{p}\left(A^{*}\right) \cap i \mathbb{R}=\emptyset$ by $\sigma_{p}\left(A_{x}^{*}\right) \cap i \mathbb{R}=\emptyset$. To characterize the latter condition in terms of the orbit through $x$ we need the following lemma.

Lemma 3.1. Let $\mathbf{T}$ be a uniformly bounded $C_{0}$-semigroup on $X$, with generator $A$, and let $x \in X$. Then $\sigma_{p}\left(A_{x}^{*}\right) \cap i \mathbb{R} \subset \sigma_{u}(A, x)$.

Proof. Let $\lambda_{0} \in \sigma_{p}\left(A_{x}^{*}\right) \cap i \mathbb{R}$. Without loss of generality we may assume $\lambda_{0}=0$. Let $0 \neq x^{*} \in D\left(A_{x}^{*}\right)$ be such that $A_{x}^{*} x^{*}=0$. Then $T_{x}^{*}(t) x^{*}=x^{*}$ for all $t \geq 0$, and hence $\left\langle x^{*}, x\right\rangle=\left\langle x^{*}, T(t) x\right\rangle$ for all $t \geq 0$. Since the linear span of the set $\{T(t) x: t \geq 0\}$ is dense in $X_{x}$, we must have $\left\langle x^{*}, x\right\rangle=: \alpha \neq 0$. Then for all $\operatorname{Re} \lambda>0$ we obtain

$$
0=\left\langle A_{x}^{*} x^{*}, R(\lambda, A) x\right\rangle=\left\langle x^{*}, A R(\lambda, A) x\right\rangle=\left\langle x^{*}, \lambda R(\lambda, A) x-x\right\rangle .
$$

Hence,

$$
\left\langle x^{*}, R(\lambda, A) x\right\rangle=\frac{1}{\lambda}\left\langle x^{*}, x\right\rangle=\frac{\alpha}{\lambda}, \quad \text { for all } \operatorname{Re} \lambda>0 .
$$

This shows that $\lim _{\lambda \downarrow 0}\|R(\lambda, A) x\|=\infty$, and hence $\lambda \mapsto R(\lambda, A) x$ has no holomorphic extension to 0 .

From this lemma we obtain the following reformulation of the condition $\sigma_{p}\left(A_{x}^{*}\right) \cap$ $i \mathbb{R}=\emptyset$ in terms of the orbit through $x$.

Proposition 3.2. Let $\mathbf{T}$ be a uniformly bounded $C_{0}$-semigroup on $X$, with generator $A$. Then for $x \in X$ the following assertions are equivalent:

(i) $\sigma_{p}\left(A_{x}^{*}\right) \cap i \mathbb{R}=\emptyset$.

(ii) $\lim _{\alpha \downarrow 0} \alpha \int_{0}^{\infty} e^{-(\alpha+\lambda) t} T(t) x d t=0$ for all $\lambda \in \sigma_{u}(A, x)$.

Proof. (i) $\Rightarrow$ (ii): Fix $\lambda \in \sigma_{u}(A, x)$. Since $\lambda \notin \sigma_{p}\left(A_{x}^{*}\right)$, the Hahn-Banach theorem implies that $D:=\left\{\left(\lambda-A_{x}\right) y: y \in D\left(A_{x}\right)\right\}$ is dense in $X_{x}$. If $\alpha>0$, then $\int_{0}^{\infty} e^{-(\alpha+\lambda) t} T(t) z d t=R(\alpha+\lambda, A) z, z \in X$, and $\sup _{\alpha>0}\|\alpha R(\alpha+\lambda, A)\|<\infty$ (see $[19,1.5 .3,1.5 .4])$. Thus for $z=\left(\lambda-A_{x}\right) y \in D$ we obtain

$$
\begin{aligned}
\lim _{\alpha \downarrow 0} \alpha \int_{0}^{\infty} e^{-(\alpha+\lambda) t} T(t) z d t & =\lim _{\alpha \downarrow 0} \alpha R(\alpha+\lambda, A)(\lambda-A) y \\
& =\lim _{\alpha \downarrow 0} \alpha y-\alpha^{2} R(\alpha+\lambda, A) y=0 .
\end{aligned}
$$

Since $(\alpha R(\alpha+\lambda, A))_{\alpha>0}$ is uniformly bounded and $D$ is dense in $X_{x}$, assertion (ii) follows.

(ii) $\Rightarrow$ (i): Suppose there is $\lambda \in \sigma_{p}\left(A_{x}^{*}\right) \cap i \mathbb{R}$ with corresponding eigenvector $0 \neq x^{*} \in D\left(A_{x}^{*}\right)$. Then $e^{-\lambda t} T_{x}^{*}(t) x^{*}=x^{*}$ for all $t \geq 0$, and $\lambda \in \sigma_{u}(A, x)$ by Lemma 
3.1. Thus

$$
\begin{aligned}
\left\langle x^{*}, x\right\rangle & =\lim _{\alpha \downarrow 0} \alpha \int_{0}^{\infty} e^{-\alpha t}\left\langle e^{-\lambda t} T_{x}^{*}(t) x^{*}, x\right\rangle d t \\
& =\lim _{\alpha \downarrow 0}\left\langle x^{*}, \alpha \int_{0}^{\infty} e^{-(\alpha+\lambda) t} T(t) x d t\right\rangle=0 .
\end{aligned}
$$

Hence $\left\langle x^{*}, T(t) x\right\rangle=\left\langle T_{x}^{*}(t) x^{*}, x\right\rangle=e^{\lambda t}\left\langle x^{*}, x\right\rangle=0$ for all $t \geq 0$. Since the linear span of $\{T(t) x: t \geq 0\}$ is dense in $X_{x}$ this implies $x^{*}=0$ which is a contradiction.

Remarks. (a) Since $\mathbf{T}$ is uniformly bounded, in assertion (ii) the convergence of the Abel means $\alpha \int_{0}^{\infty} e^{-(\alpha+\lambda) t} T(t) x d t$ as $\alpha \downarrow 0$ is equivalent to the convergence of the Cesàro means $\frac{1}{t} \int_{0}^{t} e^{-\lambda s} T(s) x d s$ as $t \rightarrow \infty$ (see [16, 2.1.5]).

(b) One always has $\sigma_{p}\left(A_{x}^{*}\right) \cap i \mathbb{R} \subset \sigma_{p}\left(A^{*}\right)$. In fact, without loss of generality let $0 \in \sigma_{p}\left(A_{x}^{*}\right) \cap i \mathbb{R}$ with corresponding eigenvector $0 \neq x^{*} \in X_{x}^{*}$. Let $z^{*} \in X^{*}$ be a Hahn-Banach extension of $x^{*}$. Then for each $t>0,\left\langle z_{t}^{*}, z\right\rangle:=$ $\frac{1}{t} \int_{0}^{t}\left\langle z^{*}, T(s) z\right\rangle d s, z \in X$, defines a linear form $z_{t}^{*} \in X^{*}$, and $\sup _{t>0}\left\|z_{t}^{*}\right\|<\infty$. If $u^{*}$ is a weak*-cluster point of $\left(z_{t}^{*}\right)_{t>0}($ as $t \rightarrow \infty)$, then $T^{*}(t) u^{*}=u^{*}$ for all $t \geq 0$ and the restriction of $u^{*}$ to $X_{x}$ coincides with $x^{*}$. Hence $A^{*} u^{*}=0$ and $u^{*} \neq 0$, i.e. $0 \in \sigma_{p}\left(A^{*}\right)$.

In order to prove our individual version of the Arendt-Batty-Lyubich-Vũ theorem we need the following construction due to Lyubich and Vũ [17]. Let $\mathbf{T}$ be a $C_{0^{-}}$ semigroup of contractions on $X$ and let $l$ be the seminorm on $X$ defined by

$$
l(x):=\lim _{t \rightarrow \infty}\|T(t) x\| .
$$

This seminorm defines a norm $l_{0}$ on the quotient space $Y_{0}:=X / \operatorname{ker} l$ by means of

$$
l_{0}(x+\operatorname{ker} l):=l(x) .
$$

Let $Y$ be the completion of $Y_{0}$ with respect to this norm. The operator $\pi: X \rightarrow Y$ given by

$$
\pi x:=x+\operatorname{ker} l
$$

is contractive and has dense range.

On $Y_{0}$ we define the linear operators $U_{0}(t)$ by $U_{0}(t) \pi x=\pi T(t) x$. These operators extend to bounded linear operators $U(t)$ on $Y$ and we have the following properties (see [17], [21, Lemma 3.1]):

(i) $\mathbf{U}=\{U(t)\}_{t \geq 0}$ is a $C_{0}$-semigroup of isometries on $Y$, and $U(t) \pi=\pi T(t)$ for all $t \geq 0$. If $B$ is the generator of $\mathbf{U}$, then $\pi D(A) \subset D(B)$ and $B \pi x=\pi A x$ for all $x \in D(A)$,

(ii) $\lim _{t \rightarrow \infty}\|T(t) x\|=\|\pi x\|$ for all $x \in X$,

(iii) $\sigma(B) \subset \sigma(A)$.

Furthermore, if $\sigma(A) \cap i \mathbb{R}$ is a proper subset of $i \mathbb{R}$, then

(iv) $\mathbf{U}$ extends to a $C_{0}$-group of isometries.

The triple $(Y, \pi, \mathbf{U})$ is called the isometric limit (semi)group associated with $\mathbf{T}$.

If $\mathbf{T}$ itself is a semigroup of isometries, then $\mathbf{U}=\mathbf{T}$ and (iv) can be written as follows (see also [3, Proposition 3.2]).

Proposition 3.3. Let $\mathbf{T}$ be a $C_{0}$-semigroup of isometries on $X$, with generator $A$. If $\sigma(A) \cap i \mathbb{R} \neq i \mathbb{R}$, then $\mathbf{T}$ extends to a group of isometries and $\sigma(A) \subset i \mathbb{R}$.

Now we come to our local version of the Arendt-Batty-Lyubich-Vũ theorem. 
Theorem 3.4. Let $\mathbf{T}$ be a uniformly bounded $C_{0}$-semigroup on a Banach space $X$, with generator $A$. Let $x \in X$ and assume that

(i) $\sigma_{u}(A, x)$ is countable,

(ii) $\lim _{\alpha \downarrow 0} \alpha \int_{0}^{\infty} e^{-(\alpha+\lambda) t} T(t) x d t=0$ for all $\lambda \in \sigma_{u}(A, x)$.

Then $\lim _{t \rightarrow \infty}\|T(t) x\|=0$.

Proof. By renorming $X$ with the equivalent norm $\|x\|:=\sup _{t \geq 0}\|T(t) x\|$ we may assume that $\mathbf{T}$ is a semigroup of contractions. Let $(Y, \pi, \mathbf{U})$ be the isometric limit semigroup associated with $\mathbf{T}$. In view of the properties of $(Y, \pi, \mathbf{U})$, the theorem is proved if we can show that $y:=\pi x=0$.

Let $F(\lambda)$ denote the holomorphic extension of $R(\lambda, A) x$ to a connected neighbourhood $V$ of $\{\operatorname{Re} \lambda \geq 0\} \backslash \sigma_{u}(A, x)$. The map $\pi F(\lambda)$ defines a holomorphic extension of $R(\lambda, B) y$ to $V$, where $B$ is the generator of $\mathbf{U}$. In particular $\sigma_{u}(B, y) \subset$ $\sigma_{u}(A, x)$. Let $Y_{y}$ be the closed linear span of the orbit $\{U(t) y: t \geq 0\}$ and let $B_{y}$ be the generator of the restriction $\mathbf{U}_{y}$ of $\mathbf{U}$ to $Y_{y}$. Then Theorem 2.2 implies that $\sigma\left(B_{y}\right) \cap i \mathbb{R}=\sigma_{u}(B, y) \subset \sigma_{u}(A, x)$, so $\sigma\left(B_{y}\right) \cap i \mathbb{R}$ is countable. On the other hand, for all $\lambda \in \sigma_{u}(B, y) \subset \sigma_{u}(A, x)$ we have

$$
\lim _{\alpha \downarrow 0} \alpha \int_{0}^{\infty} e^{-(\alpha+\lambda) t} U_{y}(t) y d t=\lim _{\alpha \downarrow 0} \pi\left(\alpha \int_{0}^{\infty} e^{-(\alpha+\lambda) t} T(t) x d t\right)=0 .
$$

Hence $\sigma_{p}\left(B_{y}^{*}\right) \cap i \mathbb{R}=\emptyset$ by Proposition 3.2. Now Theorem A applied to $\mathbf{U}_{y}$ yields $\lim _{t \rightarrow \infty}\left\|U_{y}(t) y\right\|=0$. Since $\mathbf{U}$ is a semigroup of isometries we obtain $y=\pi x=$ 0 .

As an application of Theorem 3.4 and the Remark after Proposition 3.2 we obtain the following sufficient condition for uniform stability.

Corollary 3.5. Let $\mathbf{T}$ be a uniformly bounded $C_{0}$-semigroup on a Banach space $X$, with generator A. Assume that

(i) there is a dense subspace $Z \subset X$ such that $\sigma_{u}(A, z)$ is countable for each $z \in Z$,

(ii) $\sigma_{p}\left(A^{*}\right) \cap i \mathbb{R}=\emptyset$.

Then $\mathbf{T}$ is uniformly stable.

These hypotheses are fulfilled in the following situation (see [5, Theorem 5]).

Corollary 3.6. Let $\mathbf{T}$ be a uniformly bounded $C_{0}$-semigroup on a Banach space $X$, with generator $A$. Let $\mathbf{V}$ be a $C_{0}$-semigroup on another Banach space $Z$, with generator $B$, and let $C: Z \rightarrow X$ be a bounded operator with dense range such that $T(t) C=C V(t)$ for all $t \geq 0$. If $\sigma(B) \cap i \mathbb{R}$ is countable and $\sigma_{p}\left(A^{*}\right) \cap i \mathbb{R}=\emptyset$, then $\mathbf{T}$ is uniformly stable.

Proof. By assumption, for all $z \in Z$ the map $\lambda \mapsto R(\lambda, B) z$ has a holomorphic extension $F_{z}(\lambda)$ to a neighbourhood of $\{\operatorname{Re} \lambda \geq 0\} \backslash i E$, where $i E:=\sigma(B) \cap i \mathbb{R}$ is countable. The representation of the resolvents as a Laplace transform yields $C R(\lambda, B) z=R(\lambda, A) C z$ for $\operatorname{Re} \lambda$ sufficiently large. It follows that $C F_{z}(\lambda)$ is a holomorphic extension of $R(\lambda, A) C z$ to a neighbourhood of $\{\operatorname{Re} \lambda \geq 0\} \backslash i E$. Thus, the assumptions of Corollary 3.5 are satisfied.

If the local unitary spectrum is empty we obtain the following result. 
Corollary 3.7. Let $\mathbf{T}$ be a uniformly bounded $C_{0}$-semigroup on a Banach space $X$, with generator $A$. Let $x \in X$ be such that $\sigma_{u}(A, x)=\emptyset$. Then $\lim _{t \rightarrow \infty}\|T(t) x\|=0$.

This also follows from [2, Theorem 3.5] or [2, Theorem 3.7]. By [1, Example 2.5 (b)], in Theorem 3.4 and Corollary 3.7 it is not enough to assume only boundedness of the orbit $T(\cdot) x$. However, in [7] we show for arbitrary $C_{0}$-semigroups that Theorem 3.4 is still true if the orbit is assumed to be bounded and uniformly continuous.

\section{A counterexample}

A uniformly stable semigroup $\mathbf{T}$ with generator $A$ always satisfies $\sigma_{p}\left(A^{*}\right) \cap i \mathbb{R}=$ $\emptyset$. Thus in view of Corollary 3.5 one might ask whether the existence of a dense set of vectors with countable local unitary spectrum is necessary for uniform stability of a $C_{0}$-semigroup $\mathbf{T}$. In a slightly stronger form, this was conjectured by Huang [12, p. 190].

In the following example we construct a uniformly stable $C_{0}$-semigroup such that for every non-zero vector $x$ the local unitary spectrum is the whole imaginary axis. In particular this shows that the converse of Theorem 3.4 and Corollary 3.5 is not true and gives a negative answer to the conjecture of Huang.

Example 4.1. Let $X=L^{1}\left(\mathbb{R}_{+}, w(t) d t\right)$, where $w: \mathbb{R}_{+} \rightarrow \mathbb{R}_{+}$satisfies

(i) $w$ is non-increasing,

(ii) $\lim _{t \rightarrow \infty} w(t)=0$,

(iii) for each $a>0$ there exists a constant $c>0$ such that $w(t) \geq c e^{-a t}$ for all $t \geq 0$.

Let $\mathbf{T}$ be the $C_{0}$-semigroup on $X$ defined by $T(t) f(s):=f(s-t)$ for $s \geq t \geq 0$ and 0 else, and let $A$ denote its generator. By (i) and (ii), for all $f \in X$ we have

$$
\lim _{t \rightarrow \infty}\|T(t) f\|=\lim _{t \rightarrow \infty} \int_{t}^{\infty}|f(s-t)| w(s) d s=\lim _{t \rightarrow \infty} \int_{0}^{\infty}|f(s)| w(s+t) d s=0,
$$

so $\mathbf{T}$ is uniformly stable. We will prove that 0 is the only element in $X$ whose local resolvent can be extended across some point of the imaginary axis.

By (ii), for $\operatorname{Re} \lambda<0$ the function

$$
g_{\lambda}(s):=\frac{e^{\lambda s}}{w(s)}, \quad s \geq 0,
$$

is bounded, so $h_{\lambda}(s):=e^{\lambda s}$ defines an element of $X^{*}$. For all $f \in X$ and $t \geq 0$ we have

$$
\begin{aligned}
\left\langle h_{\lambda}, T(t) f\right\rangle & =\int_{t}^{\infty} f(s-t) e^{\lambda s} d s \\
& =\int_{0}^{\infty} f(s) e^{\lambda(s+t)} d s \\
& =e^{\lambda t} \int_{0}^{\infty} f(s) e^{\lambda s} d s \\
& =e^{\lambda t}\left\langle h_{\lambda}, f\right\rangle .
\end{aligned}
$$

Thus,

$$
T^{*}(t) h_{\lambda}=e^{\lambda t} h_{\lambda}, \quad t \geq 0,
$$

so $h_{\lambda} \in D\left(A^{*}\right)$ and $A^{*} h_{\lambda}=\lambda h_{\lambda}$. 
Now suppose $f \in X$ is such that $\lambda \mapsto R(\lambda, A) f$ has a holomorphic extension $F$ to a connected neighbourhood $V$ of some point $\lambda_{0} \in i \mathbb{R}$. For $\lambda \in V$ with $\operatorname{Re} \lambda>0$,

$$
R(1, A) f=F(\lambda)+(\lambda-1) R(1, A) F(\lambda) .
$$

By analytic continuation,

$$
R(1, A) f=F(\lambda)+(\lambda-1) R(1, A) F(\lambda)=(\lambda-A) R(1, A) F(\lambda)
$$

for all $\lambda \in V$. Hence, for all $\lambda \in V$ with $\operatorname{Re} \lambda<0$,

$$
\int_{0}^{\infty} e^{\lambda s}(R(1, A) f)(s) d s=\left\langle h_{\lambda}, R(1, A) f\right\rangle=\left\langle\left(\lambda-A^{*}\right) h_{\lambda}, R(1, A) F(\lambda)\right\rangle=0 .
$$

As a function of $\lambda$, the first of these expressions is holomorphic on $\{\operatorname{Re} \lambda<0\}$ and vanishes in $\{\operatorname{Re} \lambda<0\} \cap V$. Therefore,

$$
\int_{0}^{\infty} e^{\lambda s}(R(1, A) f)(s) d s=0, \quad \text { for all } \quad \operatorname{Re} \lambda<0 .
$$

By the uniqueness of the Laplace transform, this implies that $R(1, A) f=0$. Hence $f=0$ by the injectivity of $R(1, A)$.

\section{An individual Katznelson-TZafriri theorem}

In this section we prove an individual version of the Katznelson-Tzafriri theorem. If $\mathbf{T}$ is a uniformly bounded $C_{0}$-semigroup on $X$ and $f \in L^{1}\left(\mathbb{R}_{+}\right)$then

$$
\hat{f}(\mathbf{T}) x:=\int_{0}^{\infty} f(t) T(t) x d t, \quad x \in X,
$$

defines a bounded operator $\hat{f}(T)$ on $X$. Accordingly, if $\mathbf{T}$ is a uniformly bounded $C_{0}$-group and $f \in L^{1}(\mathbb{R})$, we define an operator $\hat{f}(\mathbf{T})$ on $X$ by means of

$$
\hat{f}(\mathbf{T}) x:=\int_{-\infty}^{\infty} f(t) T(t) x d t, \quad x \in X .
$$

As usual the Fourier transform $\hat{f}$ of $f \in L^{1}(\mathbb{R})$ is given by

$$
\hat{f}(s)=\int_{-\infty}^{\infty} e^{-i s t} f(t) d t, \quad s \in \mathbb{R} .
$$

Recall that a function $f \in L^{1}(\mathbb{R})$ is of spectral synthesis with respect to a closed subset $E \subset \mathbb{R}$ if there exists a sequence $\left(f_{n}\right) \subset L^{1}(\mathbb{R})$ such that

(i) $\lim _{n \rightarrow \infty}\left\|f-f_{n}\right\|_{1}=0$,

(ii) each of the Fourier transforms $\hat{f}_{n}$ vanishes on a neighbourhood of $E$.

By regarding $L^{1}\left(\mathbb{R}_{+}\right)$as a closed subspace of $L^{1}(\mathbb{R})$, this concept extends to functions in $L^{1}\left(\mathbb{R}_{+}\right)$.

Finally we need the following well-known fact from spectral theory of isometric groups. If $\mathbf{U}$ is a $C_{0}$-group of isometries on a Banach space $X$, with generator $B$, and if $f \in L^{1}(\mathbb{R})$ is such that $\hat{f}$ vanishes in a neighbourhood of $i \sigma(B)$, then $\hat{f}(\mathbf{U})=0$ (see [11]). Thus by approximation we obtain $\hat{f}(\mathbf{U})=0$ for every $f \in L^{1}(\mathbb{R})$ which is of spectral synthesis with respect to $i \sigma(B)$.

Now we can show the following individual version of Theorem B. 
Theorem 5.1. Let $\mathbf{T}$ be a uniformly bounded $C_{0}$-semigroup on a Banach space $X$, with generator $A$, and let $x \in X$. Then

$$
\lim _{t \rightarrow \infty}\|T(t) \hat{f}(\mathbf{T}) x\|=0
$$

for each $f \in L^{1}\left(\mathbb{R}_{+}\right)$which is of spectral synthesis with respect to $i \sigma_{u}(A, x)$.

Proof. Let $f \in L^{1}\left(\mathbb{R}_{+}\right)$be of spectral synthesis with respect to $i \sigma_{u}(A, x)$. We may assume that $i \sigma_{u}(A, x)$ is a proper subset of $\mathbb{R}$ (otherwise $\hat{f}=0$, and hence $f=0)$. By renorming $X$ we can assume that $\mathbf{T}$ is a semigroup of contractions. Let $(Y, \pi, \mathbf{U})$ be the isometric limit semigroup associated with $\mathbf{T}$ and let $B$ be the generator of $\mathbf{U}$. If $y:=\pi x$ then $\sigma_{u}(B, y) \subset \sigma_{u}(A, x)$. Let $Y_{y}$ be the closed linear span of the orbit of $\mathbf{U}$ through $y$ and let $B_{y}$ be the generator of the restriction $\mathbf{U}_{y}$ of $\mathbf{U}$ to $Y_{y}$. Theorem 2.2 implies that $\sigma\left(B_{y}\right) \cap i \mathbb{R}=\sigma_{u}(B, y) \subset \sigma_{u}(A, x)$. By Proposition 3.3, $\mathbf{U}_{y}$ extends to a group of isometries (which we denote again by $\mathbf{U}_{y}$ ) and $\sigma\left(B_{y}\right) \subset \sigma_{u}(A, x)$. Thus our above discussion yields $\hat{f}\left(\mathbf{U}_{y}\right)=0$. In particular, $0=\hat{f}\left(\mathbf{U}_{y}\right) \pi x=\pi \hat{f}(\mathbf{T}) x$, i.e. $\lim _{t \rightarrow \infty}\|T(t) \hat{f}(\mathbf{T}) x\|=0$.

\section{Countable local spectrum and Relative Compactness of an orbit}

A $C_{0}$-semigroup $\mathbf{T}$ on $X$ is called (weakly) almost periodic if for each $x \in X$ the orbit $\{T(t) x: t \geq 0\}$ is relatively (weakly) compact. Lyubich and Vũ $[22$, Theorem $2]$ have shown that if in Theorem A the assumption $\sigma_{p}\left(A^{*}\right) \cap i \mathbb{R}$ is replaced by a certain ergodic condition on $\mathbf{T}$, then the semigroup $\mathbf{T}$ is almost periodic (see also [8, Theorem 8]). More precisely, the following holds.

Let $\mathbf{T}$ be a uniformly bounded $C_{0}$-semigroup on a Banach space $X$, with generator A. If $\sigma(A) \cap i \mathbb{R}$ is countable, then the following assertions are equivalent:

(i) $\mathbf{T}$ is almost periodic.

(ii) $\mathbf{T}$ is weakly almost periodic.

(iii) $\lim _{\alpha \downarrow 0} \alpha \int_{0}^{\infty} e^{-(\alpha+\lambda) t} T(t) x d t$ exists for all $x \in X$ and all $\lambda \in i \mathbb{R}$.

It is easy to see that in (iii) it suffices to require the convergence of the Abel means $\alpha \int_{0}^{\infty} e^{-(\alpha+\lambda) t} T(t) x d t$ as $\alpha \rightarrow 0$ only for $\lambda \in \sigma(A) \cap i \mathbb{R}$. The previous result has the following individual version.

Theorem 6.1. Let $\mathbf{T}$ be a uniformly bounded $C_{0}$-semigroup on a Banach space $X$, with generator $A$. Let $x \in X$ be such that $\sigma_{u}(A, x)$ is countable. Then the following assertions are equivalent:

(i) The set $\{T(t) x: t \geq 0\}$ is relatively compact.

(ii) The set $\{T(t) x: t \geq 0\}$ is relatively weakly compact.

(iii) $\lim _{\alpha \downarrow 0} \alpha \int_{0}^{\infty} e^{-(\alpha+\lambda) t} T(t) x d t$ exists for all $\lambda \in \sigma_{u}(A, x)$.

Proof. Clearly (i) $\Rightarrow$ (ii), and (ii) $\Rightarrow$ (iii) is a well-known result from ergodic theory (see $[16,2.1 .5])$.

(iii) $\Rightarrow$ (i): By renorming $X$ we may assume that $\mathbf{T}$ is a semigroup of contractions. Let $X_{c} \subset X$ be the closed subspace of all vectors $z \in X$ having a relatively compact orbit $\{T(t) z: t \geq 0\}$. The space $X_{c}$ is invariant under $\mathbf{T}$. If $q: X \rightarrow X / X_{c}$ is the quotient map, then $\mathbf{T}$ induces a $C_{0}$-semigroup of contractions $\mathbf{T}_{/}=\left\{T_{/}(t)\right\}_{t \geq 0}$ on $X / X_{c}$ by means of $T_{/}(t) q(z):=q(T(t) z), z \in X$ (see [18, A-I.3.3]). The generator 
$A_{\text {/ of }} \mathbf{T}_{/}$satisfies $R\left(\lambda, A_{/}\right) q=q R(\lambda, A)$ for $\operatorname{Re} \lambda>0$. Thus

$$
\sigma_{u}(A /, q x) \subset \sigma_{u}(A, x) .
$$

On the other hand if $\lambda \in \sigma_{u}(A, x)$, then $x_{\lambda}:=\lim _{t \rightarrow \infty} \frac{1}{t} \int_{0}^{t} e^{-\lambda s} T(s) x d s$ satisfies $T(t) x_{\lambda}=e^{\lambda t} x_{\lambda}$ for all $t \geq 0$. Thus $x_{\lambda} \in X_{c}$, and hence

$$
\lim _{\alpha \downarrow 0} \alpha \int_{0}^{\infty} e^{-(\alpha+\lambda) t} T_{/}(t) q x d t=q\left(\lim _{\alpha \downarrow 0} \alpha \int_{0}^{\infty} e^{-(\alpha+\lambda) t} T(t) x d t\right)=0
$$

for all $\lambda \in \sigma_{u}(A /, q x) \subset \sigma_{u}(A, x)$. Now we can apply Theorem 3.4 to $\mathbf{T} /$ and $q x$ and obtain

$$
\lim _{t \rightarrow \infty}\left\|T_{/}(t) q x\right\|=0 .
$$

For fixed $\epsilon>0$ we can find $t_{0} \geq 0$ and $z \in X_{c}$ such that $\left\|T\left(t_{0}\right) x-z\right\|<\epsilon$. Thus $\left\|T\left(t+t_{0}\right) x-T(t) z\right\|<\epsilon$ for all $t \geq 0$. If $B_{X}:=\{x \in X:\|x\| \leq 1\}$ denotes the unit ball of $X$, then

$$
\{T(t) x: t \geq 0\} \subset C_{\epsilon}+\epsilon B_{X},
$$

where $C_{\epsilon}:=\left\{T(t) x: 0 \leq t \leq t_{0}\right\} \cup \overline{\{T(t) z: t \geq 0\}}$ is compact. Since this holds for every $\epsilon>0$ the orbit $\{T(t) x: t \geq 0\}$ is precompact, and hence relatively compact.

The uniform boundedness assumption cannot be weakened to boundedness of $T(\cdot) x$, as is shown by the example mentioned at the end of Section 3.

\section{A QUANTITATIVE INDIVIDUAL STABILITy RESUlt}

In [6, Theorem 5.3] it was proved that if $\mathbf{T}$ is a semigroup of contractions such that $\sigma(A) \cap i \mathbb{R}$ is countable, then

$$
\lim _{t \rightarrow \infty}\|T(t) x\|=\inf \left\{\|x-z\|: z \in X_{0}\right\}, \quad x \in X,
$$

where $X_{0}=\left\{z \in X: \lim _{t \rightarrow \infty}\|T(t) z\|=0\right\}$. If $\mathbf{T}$ is trivially asymptotically stable, i.e. $X_{0}=\{0\}$, then $\mathbf{T}$ extends to a group of isometries (see [6, Theorem 4.8]). In this section, we apply our previous results to obtain an analogue for an individual orbit.

First we recall the following notion from harmonic analysis. A closed set $E \subset \mathbb{R}$ is a set of spectral synthesis if every function $f \in L^{1}(\mathbb{R})$ whose Fourier transform $\hat{f}$ vanishes on $E$ is of spectral synthesis with respect to $E$ (see Section 5 ). It is well-known that every countable closed set in $\mathbb{R}$ is a set of spectral synthesis (see $[14$, VIII.7.3]).

The following lemma is a local version of [6, Proposition 4.6].

Lemma 7.1. Let $\mathbf{T}$ be a trivially asymptotically stable $C_{0}$-semigroup of contractions on $X$, with generator $A$. Let $x \in X$ be such that $\sigma_{u}(A, x)$ is countable and the linear span of the orbit through $x$ is dense in $X$. Then there is a contractive algebra homomorphism $\xi: L^{1}(\mathbb{R}) \rightarrow L(X)$ with the following properties:

(i) $\xi(f)=\hat{f}(\mathbf{T})$ for all $f \in L^{1}\left(\mathbb{R}_{+}\right)$,

(ii) $\xi\left(g_{t}\right)=T(t) \xi(g)$ for all $g \in L^{1}(\mathbb{R})$ and $t \geq 0$, where $g_{t}:=g(\cdot-t)$,

(iii) if $\hat{g} \equiv 0$ on $i \sigma_{u}(A, x)$ for $g \in L^{1}(\mathbb{R})$, then $\xi(g)=0$. 
Proof. Let $(Y, \pi, \mathbf{U})$ be the isometric limit semigroup associated with $\mathbf{T}$ and let $B$ be the generator of $\mathbf{U}$. The linear span of the orbit of $\mathbf{U}$ through $\pi x$ is dense in $Y$. Thus Theorem 2.2 implies that $E:=i \sigma(B) \cap \mathbb{R}=i \sigma_{u}(B, x) \subset i \sigma_{u}(A, x)$ is countable. By property (iv) of the isometric limit semigroup (see Section 3) $\mathbf{U}$ extends to a group of isometries which we denote again by $\mathbf{U}$. In particular, $i \sigma(B)=i \sigma(B) \cap \mathbb{R}=E$.

Let $f \in L^{1}(\mathbb{R})$. Since $E$ is countable, a result of Esterle, Strouse and Zouakia [10, Lemme 3.8] (see also [6, Proposition 2.1], or [13, Théorème 2] for the discrete case) implies that for each $\epsilon>0$ there exists $h \in L^{1}\left(\mathbb{R}_{+}\right)$such that $\left.\hat{h}\right|_{E}=\left.\hat{f}\right|_{E}$ and $\|h\|_{1} \leq(1+\epsilon)\|f\|_{1}$. Again by the countability of $E$ we obtain that $h-f$ is of spectral synthesis with respect to $E$. Then $(h-f)^{\wedge}(\mathbf{U})=0$ (see Section 5), i.e. $\hat{f}(\mathbf{U})=\hat{h}(\mathbf{U})$. Thus $\hat{f}(\mathbf{U}) \pi=\hat{h}(\mathbf{U}) \pi=\pi \hat{h}(\mathbf{T})$, and hence $\hat{f}(\mathbf{U}) \pi z \in \pi X$ for all $z \in X$. Since $\mathbf{T}$ is trivially asymptotically stable, $\pi: X \rightarrow Y$ is injective. So we may define

$$
\xi(f) z:=\pi^{-1} \hat{f}(\mathbf{U}) \pi z=\hat{h}(\mathbf{T}) z, \quad z \in X .
$$

Then $\|\xi(f) z\|=\|\hat{h}(\mathbf{T}) z\| \leq\|h\|_{1}\|z\| \leq(1+\epsilon)\|f\|_{1}\|z\|$ for $z \in X$. So $\xi: L^{1}(\mathbb{R}) \rightarrow$ $L(X)$ is a contraction. It follows directly from the construction that $\xi$ is an algebra homomorphism and that (i) and (ii) hold.

Let $g \in L^{1}(\mathbb{R})$ be such that $\hat{g}$ vanishes on $i \sigma_{u}(A, x) \supset i \sigma(B)=E$. Since $E$ is countable it is a set of spectral synthesis, and as above we obtain that $\hat{g}(\mathbf{U})=0$. Thus $\xi(g)=\pi^{-1} \hat{g}(\mathbf{U}) \pi=0$, which proves property (iii).

For a given $C_{0}$-semigroup $\mathbf{T}$ on $X$ and $x \in X$, a map $\eta: \mathbb{R} \rightarrow X$ is called a complete orbit through $x$ if

(i) $\eta(0)=x$,

(ii) $T(t) \eta(s)=\eta(t+s)$ for all $t \geq 0$ and $s \in \mathbb{R}$.

It follows from (i) and (ii) that $\eta(t)=T(t) x$ for all $t \geq 0$, so $\eta$ is an extension of $T(\cdot) x$ to negative $t$.

Now we are in a position to prove the main result of this section. It is a local version of [6, Theorem 4.8]. Our argument simplifies the proof given there, so that we feel justified in presenting it in detail. Recall that $\mathbf{T}_{x}$ is the restriction of $\mathbf{T}$ to the closed linear span $X_{x}$ of the orbit through $x$.

Theorem 7.2. Let $\mathbf{T}$ be a trivially asymptotically stable $C_{0}$-semigroup of contractions on $X$, with generator $A$. Let $x \in X$ be such that $\sigma_{u}(A, x)$ is countable. Then $\mathbf{T}_{x}$ extends to a $C_{0}$-group of isometries. In particular for every $z \in X_{x}$ there is a complete isometric orbit through z.

Proof. By the Remark after Theorem 2.2 we have $\sigma_{u}(A, x)=\sigma_{u}\left(A_{x}, x\right)$. Thus without loss of generality we may assume that $X=X_{x}$.

Fix $z \in X$. Let $f \in L^{1}(\mathbb{R})$ be such that its Fourier transform has compact support $K$. For $t \in \mathbb{R}$ we define

$$
\eta_{f}(t):=\xi\left(f_{t}\right) z
$$

where $\xi$ is given as in the previous lemma and $f_{t}:=f(\cdot-t)$. Fix $\epsilon>0$. By a well-known result from harmonic analysis (see $[20,2.6 .8]$ ) we can choose $h \in L^{1}(\mathbb{R})$ such that $\hat{h} \equiv 1$ on $K$ and $\|h\|_{1} \leq 1+\epsilon$. Then $\hat{h} \hat{f}=\hat{f}$ and

$$
\eta_{f}(t)=\xi\left(f_{t}\right) z=\xi\left((h * f)_{t}\right) z=\xi\left(h_{t} * f\right) z=\xi\left(h_{t}\right) \xi(f) z, \quad t \in \mathbb{R},
$$


where $*$ denotes the convolution. So

$$
\sup _{t \in \mathbb{R}}\left\|\eta_{f}(t)\right\| \leq(1+\epsilon)\|\xi(f) z\| .
$$

Lemma 7.1 implies $\eta_{f}(t)=\xi\left(f_{t}\right) z=T(t) \xi(f) z=T(t) \eta_{f}(0)$ for $t \geq 0$. Hence $\eta_{f}$ is a complete bounded orbit through $\eta_{f}(0)=\xi(f) z$ and

$$
\sup _{t \in \mathbb{R}}\left\|\eta_{f}(t)\right\|=\|\xi(f) z\| .
$$

Since $\mathbf{T}$ is trivially asymptotically stable $\eta_{f}$ is the unique extension of $T(\cdot) \xi(f) z$ to a complete bounded orbit.

We claim that there is a sequence $\left(f_{n}\right) \subset L^{1}(\mathbb{R})$ such that the Fourier transform of each $f_{n}$ is compactly supported and

$$
\lim _{n \rightarrow \infty}\left\|z-\xi\left(f_{n}\right) z\right\|=0 .
$$

There is a slight abuse of notation here; the subscript $n$ indexes the sequence $\left(f_{n}\right)$ and does not mean the translate of $f$ with respect to $n$.

To prove the claim, fix $\epsilon>0$. By taking a suitable non-negative function supported in a small interval $[0, \delta]$ we can find an $h \in L^{1}\left(\mathbb{R}_{+}\right)$with $\|h\|_{1}=1$ such that $\|z-\hat{h}(\mathbf{T}) z\| \leq \epsilon / 2$. Since the functions whose Fourier transform has compact support are dense in $L^{1}(\mathbb{R})$ (see [14, VI.1.12]) we can find $g \in L^{1}(\mathbb{R})$ such that $\hat{g}$ has compact support and $\|h-g\|_{1} \leq \epsilon /(2+\|z\|)$. Then

$$
\begin{aligned}
\|z-\xi(g) z\| & \leq\|\xi(h-g) z\|+\|z-\xi(h) z\| \\
& \leq\|h-g\|_{1}\|z\|+\|z-\hat{h}(\mathbf{T}) z\| \leq \epsilon .
\end{aligned}
$$

This proves the claim.

For each $n$, let $\eta_{n}(\cdot)$ denote the unique complete orbit through $\xi\left(f_{n}\right) z$ constructed above. Then $\eta_{n}(\cdot)-\eta_{m}(\cdot)$ is the unique complete orbit through $\xi\left(f_{n}-f_{m}\right) z$ and hence

$$
\sup _{t \in \mathbb{R}}\left\|\eta_{n}(t)-\eta_{m}(t)\right\|=\left\|\xi\left(f_{n}-f_{m}\right) z\right\|=\left\|\xi\left(f_{n}\right) z-\xi\left(f_{m}\right) z\right\| .
$$

Noting that the right hand side tends to 0 as $n, m \rightarrow \infty$, we can define $z(t):=$ $\lim _{n \rightarrow \infty} \eta_{n}(t)$. Then $z(\cdot)$ is a complete bounded orbit through $\lim _{n \rightarrow \infty} \eta_{n}(0)=$ $\lim _{n \rightarrow \infty} \xi\left(f_{n}\right) z=z$ and

$$
\sup _{t \in \mathbb{R}}\|z(t)\| \leq \limsup _{n \rightarrow \infty}\left\|\xi\left(f_{n}\right) z\right\|=\|z\| .
$$

Define

$$
U(t) z:=z(t), \quad t \in \mathbb{R} .
$$

Then $U(t)$ is a well-defined linear operator on $X$. Morover $U(t) U(-t)=I$ and $\|U(t)\| \leq 1$ for $t \in \mathbb{R}$, and $U(t)=T(t)$ for $t \geq 0$. It follows that $\mathbf{U}$ is an isometric group extending $\mathbf{T}$.

If $\mathbf{T}$ is a semigroup of contractions we can use the previous theorem to obtain a formula for the $\operatorname{limit}_{t \rightarrow \infty}\|T(t) x\|$. For this we need the following lemma which is proved in [6, Proposition 4.3]. Recall that $X_{0}=\left\{z \in X: \lim _{t \rightarrow \infty}\|T(t) z\|=0\right\}$.

Lemma 7.3. Let $\mathbf{T}$ be a $C_{0}$-semigroup of contractions on $X$ and let $\mathbf{T} /$ be the quotient semigroup on $X_{/}:=X / X_{0}$. Then for all $x \in X$,

$$
\lim _{t \rightarrow \infty}\left\|T_{/}(t)\left(x+X_{0}\right)\right\|=\lim _{t \rightarrow \infty}\|T(t) x\| .
$$


In particular, $\mathbf{T}_{/}$is trivially asymptotically stable.

Now we come to the announced local version of [6, Theorem 5.3].

Theorem 7.4. Let $\mathbf{T}$ be a $C_{0}$-semigroup of contractions on a Banach space $X$, with generator $A$. Let $x \in X$ be such that $\sigma_{u}(A, x)$ is countable. Then

$$
\lim _{t \rightarrow \infty}\|T(t) x\|=\inf \left\{\|x-z\|: z \in X_{0}\right\} .
$$

Proof. Let $Z:=X_{x}$ be the closed linear span of the orbit through $x$ and $\mathbf{S}:=\mathbf{T}_{x}$ the restriction of $\mathbf{T}$ to $Z$, with generator $B$. Then $\sigma_{u}(A, x)=\sigma_{u}(B, x)$ (see the remark after Theorem 2.2). Denote by $\mathbf{S}$, the quotient semigroup on $Z /=Z / Z_{0}$, where $Z_{0}:=\left\{z \in Z: \lim _{t \rightarrow \infty}\|S(t) z\|=0\right\} \subset X_{0}$. Lemma 7.3 implies that $\mathbf{S} /$ is a trivially asymptotically stable $C_{0}$-semigroup of contractions. If $B /$ is the generator of $\mathbf{S}_{/}$and $q: Z \rightarrow Z$ / is the quotient map, then $R\left(\lambda, B_{/}\right) q=q R(\lambda, B)$ for all $\operatorname{Re} \lambda>0$ (see [18, A-III.4.2]). Thus $\sigma_{u}(B /, q x) \subset \sigma_{u}(B, x)$. Finally, the linear span of the orbit of $\mathbf{S}$ / through $q x$ is dense in $Z_{/}$. Hence, by Theorem $7.2, \mathbf{S}$ / extends to a group of isometries, and by Lemma 7.3,

$$
\begin{aligned}
\lim _{t \rightarrow \infty}\|T(t) x\| & =\lim _{t \rightarrow \infty}\left\|S_{/}(t)\left(x+Z_{0}\right)\right\|=\left\|x+Z_{0}\right\| \\
& \geq\left\|x+X_{0}\right\| .
\end{aligned}
$$

On the other hand, since $\mathbf{T}$ is contractive, for all $z \in X_{0}$ we have

$$
\lim _{t \rightarrow \infty}\|T(t) x\|=\lim _{t \rightarrow \infty}\|T(t)(x-z)\| \leq\|x-z\| .
$$

Taking the infimum over all $z \in X_{0}$, it follows that

$$
\lim _{t \rightarrow \infty}\|T(t) x\| \leq\left\|x+X_{0}\right\| .
$$

Hence

$$
\lim _{t \rightarrow \infty}\|T(t) x\|=\left\|x+X_{0}\right\|=\inf \left\{\|x-z\|: z \in X_{0}\right\}
$$

\section{ACKNOWLEDGEMENT}

The semigroup of Example 4.1 was suggested to us by Ralph deLaubenfels as a possible candidate for a counterexample.

\section{REFERENCES}

1. W. Arendt and C.J.K. Batty, Tauberian theorems and stability of one-parameter semigroups, Trans. Amer. Math. Soc. 306 (1988), 837-852. MR 89g:47053

2. W. Arendt and J. Prüss, Vector-valued Tauberian theorems and asymptotic behavior of linear Volterra equations, SIAM J. Math. Anal. 23 (1992), 412-448. MR 92m:47150

3. C.J.K. Batty, Asymptotic behaviour of semigroups of operators, Functional analysis and operator theory (J. Zemánek, ed.), vol. 30, Banach Center Publ., Warsaw, 1994, pp. 35-52. MR 95g:47058

4. C.J.K. Batty, Tauberian theorems for the Laplace-Stieltjes transform, Trans. Amer. Math. Soc. 322 (1990), 783-804. MR 91c:44001

5. C.J.K. Batty, Spectral conditions for stability of one-parameter semigroups, J. Diff. Equations 127 (1996), 87-96. MR 97e:47060

6. C.J.K. Batty, Z. Brzeźniak and D.A. Greenfield, A quantitative asymptotic theorem for contraction semigroups with countable unitary spectrum, Studia Math. 121 (1996), 167-183. CMP 97:03

7. C.J.K. Batty, J. van Neerven and F. Räbiger, Tauberian theorems and stability of solutions of the Cauchy problem, Trans. Amer. Math. Soc. 350 (1998), 2087-2103. 
8. C.J.K. Batty and Vũ Quôc Phóng, Stability of individual elements under one-parameter semigroups, Trans. Amer. Math. Soc. 322 (1990), 805-818. MR 91c:47072

9. R. deLaubenfels and Vũ Quôc Phóng, Stability and almost periodicity of solutions of ill-posed abstract Cauchy problems, Proc. Amer. Math. Soc. 125 (1997), 235-241. MR 97c:34123

10. J. Esterle, E. Strouse and F. Zouakia, Stabilité asymptotique de certains semigroupes d'opérateurs et idéaux primaires de $L^{1}\left(\mathbb{R}_{+}\right)$, J. Operator Theory 28 (1992), 203-227. MR 95f: 43001

11. D.E. Evans, On the spectrum of a one-parameter strongly continuous representation, Math. Scand. 39 (1976), 80-82. MR 55:3873

12. Falun Huang, Spectral properties and stability of one-parameter semigroups, J. Diff. Equations 104 (1993), 182-195. MR 94f:47047

13. J.P. Kahane and Y. Katznelson, Sur les algèbres de restrictions des séries de Taylor absolument convergentes à un fermé du cercle, J. Analyse Math. 23 (1970), 185-197. MR 42:8179

14. Y. Katznelson, An Introduction to Harmonic Analysis, Dover Publications, 2nd ed., New York, 1976. MR 54:10976

15. Y. Katznelson and L. Tzafriri, On power bounded operators, J. Functional Anal. 68 (1986), 313-328. MR 88e:47006

16. U. Krengel, Ergodic theorems, De Gruyter, Berlin, 1985. MR 87i:28001

17. Yu. I. Lyubich and Vũ Quôc Phóng, Asymptotic stability of linear differential equations on Banach spaces, Studia Math. 88 (1988), 37-42. MR 89e:47062

18. R. Nagel (ed.), One-parameter Semigroups of Positive Operators, Springer Lect. Notes in Math. 1184, 1986. MR 88i:47022

19. A. Pazy, Semigroups of Linear Operators and Applications to Partial Differential Equations, Springer-Verlag, New York, Berlin, Heidelberg, Tokyo, 1983. MR 85g:47061

20. W. Rudin, Fourier Analysis on Groups, Interscience, New York, 1962. MR 27:2808

21. Vũ Quôc Phóng, Theorems of Katznelson-Tzafriri type for semigroups of operators, J. Functional Anal. 103 (1992), 74-84. MR 93e:47050

22. Vũ Quôc Phóng and Yu.I. Lyubich, A spectral criterion for asymptotic almost periodicity of uniformly continuous representations of abelian semigroups, J. Soviet Math. 48 (1990), 644-647. MR 90e:22004 (Russian original)

St. John's College, Oxford OX1 3JP, England

E-mail address: charles.batty@sjc.ox.ac.uk

Department of Mathematics, Delft Technical University, P. O. Box 356, 2600 AJ Delft, The Netherlands

E-mail address: J.vanNeerven@twi.tudelft.nl

Mathematisches Institut, Universität Tübingen, Auf der Morgenstelle 10, D-72076 Tübingen, Germany

E-mail address: frra@michelangelo.mathematik.uni-tuebingen.de 